\title{
Experimental cryo-irrigation of the knee joint
}

\author{
S C CHEN, B HELAL, P A REVELL, R BROCKLEHURST, AND
}

H L F CURREY

From the Bone and Joint Research Unit, The London Hospital Medical College, London; and Highlands Hospital, Winchmore Hill, London

SUMMARY Experiments have been carried out to test the feasibility of using cryo-irrigation as a means of ablating the synovium in the rheumatoid knee joint. Cryo-irrigation was performed by a cooling machine and pump, which circulated cold 200/10 centistoke (cSt) silicone through the knee joint of rabbits anaesthetised with intravenous (IV) 'Saffan'. Fluid left the joint at -5 to $-10^{\circ} \mathrm{C}$. Sixteen normal New Zealand rabbits received cryo-irrigation of one knee joint for $10-20$ minutes and were killed at one day, and one, two, and 12 weeks thereafter. Judged by radioactive sulphate incorporation there was no impairment of chondrocyte function in the articular cartilage of irrigated joints. Histological examination showed mild synovitis and some loss of staining of superficial cartilage in $6 / 16$ irrigated joints ( $v 1 / 16$ control joints). Similar treatment of rabbit joints in which the Glynn model of synovitis had been induced showed marked reduction of synovitis 14-45 days after silicone treatment. Nine of 26 animals in which synovitis was induced in both knees and cryo-irrigation performed in one knee died either immediately postoperatively or during the next week. These deaths remain unexplained. A single dog received cryo-irrigation of one knee $\left(-6\right.$ to $-9^{\circ} \mathrm{C}$ for $\left.22 \mathrm{~min}\right)$ and remained perfectly well up to sacrifice at six months, when the joint appeared histologically completely normal.

Key words: silicone, synovitis, rheumatoid arthritis, rabbits, dogs, radioactive sulphate, histology.

Clear evidence points to the proliferated synovial membrane playing a central part in the destructive joint changes of rheumatoid arthritis. Predictably, attempts have been made to treat the disease by removal or ablation of this structure with surgical, chemical, or radioisotopic methods. The results have been somewhat disappointing, though short term symptomatic improvement has tended to divert attention from the failure to influence the longer term outcome. In addition, injected chemicals and radioisotopes may produce undesirable side effects both locally on cartilage and on remote structures. Nevertheless, at least in theory, local treatment remains an attractive approach to the management of the rheumatoid knee with gross synovial proliferation.

Technical developments in cryosurgery now make it feasible to irrigate deep structures with fluid at

Accepted for publication 7 April 1986.

Correspondence to $\mathrm{Mr} \mathrm{S} \mathrm{C}$ Chen, Enfield District Hospital, Highlands Wing, Winchmore Hill, London N21 1PN. temperatures sufficiently low to cause cell death and tissue necrosis-without the hazards of chemical or radiation toxicity. Further, the synovial cavity provides a space suitable for cryo-irrigation, with some prospect of achieving necrosis of the surface layers. Clearly, this would expose articular cartilage also to cold, and if this technique were to be tested clinically it would be necessary first to establish the risk of cartilage damage.

This study was undertaken to examine the feasibility of cryo-irrigation of the rabbit knee joint, and to test the effect of this both on cartilage viability and on experimental immune synovitis. One longer term experiment was also carried out in a dog.

\section{Materials and methods}

TREATMENT OF ANIMALS

New Zealand white rabbits weighing approximately $3 \mathrm{~kg}$ were housed in individual cages. The experiments were performed in three stages as follows: 


\section{Experiment 1}

Seventeen normal rabits received a cold silicone perfusion (200/10 cSt) into one knee. One animal suffered sudden cardiac arrest during the procedure, leaving 16 available for histological studies at one day and one, two, and 12 weeks after perfusion.

\section{Experiment 2}

Six normal rabbits were perfused with silicone of different viscosities (two each of 200/1, 200/10, and $200 / 200 \mathrm{cSt}$ ) at ambient temperature in order to assess the effects of this material without cooling. All six were killed after five weeks for histological examination of the joints.

\section{Experiment 3}

Twenty six rabbits were sensitised with bovine serum albumin (BSA), then perfused with cold silicone into one knee five weeks after the induction of synovitis by intra-articular injection (see below). Nine of these animals died of sudden cardiac arrest during the cold silicione perfusion procedure, but no cause for this was found at autopsy in any of them. The remaining 17 animals were killed one day and one, two, and four weeks after perfusion, when both knees were taken for histological examination.

\section{PERFUSION OF KNEE JOINTS WITH COLD SILICONE}

The cooling machine, developed to allow perfusion of joints with cold silicone, was prepared one hour in advance by filling the sterile closed circuit system, including a one litre capacity bag, with silicone fluid (200/10 cSt; Dow Corning), cooling to $-20^{\circ} \mathrm{C}$, and circulating the silicone with the pump (Fig. 1). The rabbits were anaesthetised with Saffan (Glaxovet) $(9 \mathrm{mg} / \mathrm{kg}, \mathrm{IV})$, and one knee was irrigated with cooled silicone at -5 to $-10^{\circ} \mathrm{C}$ for between 10 and $20 \mathrm{~min}$ by means of inlet and outlet needles. The former needle was slightly smaller than the latter to avoid an undue rise of pressure within the joint. The temperature at the inlet and outlet was monitored constantly throughout the procedure. Silicone was removed from the joint by digital pressure at the end $\frac{}{n}$. of the perfusion period and the animals allowed full freedom of movement postoperatively.

IN DUCTION OF SYNOVITIS

Each animal was immunised with BSA (Sigma) $5 \mathrm{mg}$ 离 dissolved in $1 \mathrm{ml}$ water and emulsified with an equal $\overline{0}$ volume of Freund's complete adjuvant (FCA) Difco). BSA/FCA emulsion $(2 \mathrm{ml})$ was injected in- ${ }^{\infty}$ tradermally divided between multiple sites over the $\vec{\circ}$ back. Three weeks later $2.5 \mathrm{mg}$ BSA dissolved in $0.5 \overrightarrow{\vec{H}}$ $\mathrm{ml}$ physiological saline, after passage through $\mathrm{a}_{\odot}^{\omega}$ Millipore filter $(0.45 \mu \mathrm{m}$ pore size), was injected into each knee joint with aseptic precautions, and? using an anterior approach through the patellarct ligament. Two weeks later these intra-articular $\overrightarrow{0}$ injections were repeated. Cold perfusion of oneo knee joint was carried out five weeks after the or second intra-articular injection.

INCORPORATION OF RADIOACTIVE SULPHUR@ INTO CARTILAGE

Articular cartilage was shaved off the femoral condyles with a scalpel. Each slice was about $1 \mathrm{~mm} \overrightarrow{\vec{O}}$ thick and measured about $2 \mathrm{~mm}$ square. These shavings were placed in vials containing $5 \mathrm{ml}$ sterife? Eagle's medium and allowed to equilibrate wishio $\left[{ }^{35}\right.$ S $]$ sulphate and nutrients from the medium at $4 . C$ for 20 minutes. The vials were then incubated ato $37^{\circ} \mathrm{C}$ for a measured period, usually four hours. Next the cartilage was equilibrated in four changes $\stackrel{\AA}{\varrho}$ of $0 \cdot 15 \mathrm{M} \mathrm{NaCl}(20 \mathrm{ml})$ at $4^{\circ} \mathrm{C}$ for 30 minutes on $\overrightarrow{0}$ each occasion. A final, fifth, wash $(2 \mathrm{ml})$, also 3 lasting 30 minutes, was checked for $\left[{ }^{35} \mathrm{~S}\right]$ sulphate labelled material. None was detected. Cartilage was? stored at $-20^{\circ} \mathrm{C}$ while awaiting radioisotopic measurement. Rates of sulphate incorporation were calcu- 0 lated as millimoles per hour per gram of wet tissue.

\section{H IS T OLOGICAL EXAMINATION}

Both knees were fixed in $10 \%$ formal saline, decalcified in $5 \%$ formic acid/formalin, and divided $\stackrel{\circ}{-}$ so as to enable examination of the femora, tibiae,

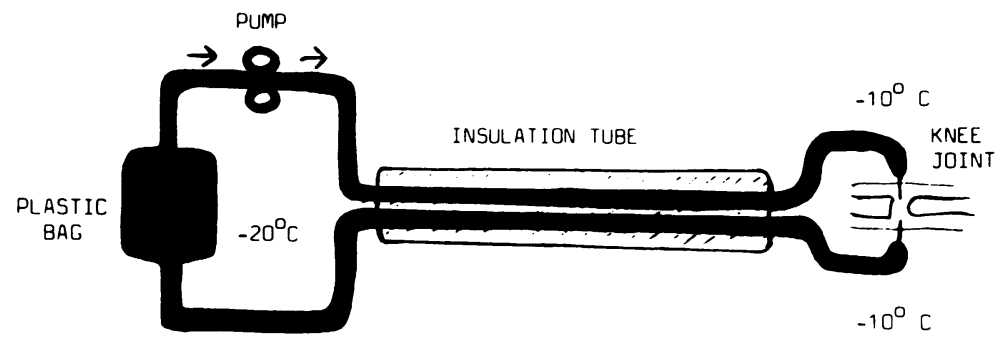

Fig. 1 Diagrammatic representation of the cooling circuit. 
and patellae. After paraffin wax embedding $7 \mu \mathrm{m}$ sections were cut and stained with haematoxylin and eosin. The histological examination was performed without knowledge of the treatment received by a particular joint. In experiment 1 the right knee was injected with cold silicone in all but five animals, but since the pathologist had no difficulty in detecting the synovial response to silicone further variation of the side receiving cold treatment became pointless, and all subsequent animals were perfused experimentally on the right side. Information about viscosity of the silicone and the precise treatment regimen in the sensitised animals was withheld from the pathologist.

\section{Results}

NORMAL JOINTS PERFUSED WITH COLD S I L I C ONE

\section{Histological appearances}

A total of 16 animals was available for studies of the effects of cold silicone. Three were killed at one day, two at one week, eight at two weeks, and three at 12 weeks after perfusion. The synovium from the joint receiving cold silicone treatment showed intimal cell hyperplasia with a predominance of macrophages and occasional giant cells making up the underlying infiltrate in the synovial connective tissues in relation to spaces representing the sites of silicone deposition (Fig. 2). Polymorphonuclear leucocytes were present in two out of three synovia one day after perfusion but were not seen in the rest of the joints. No evidence of necrosis of the synovium was seen in any of these silicone injected joints. The contralateral uninjected joints were normal in all the animals with one exception, in which there were polymorphonuclear leucocytes and some lymphocytes present one day after experimentation on the other side.

The articular cartilage showed loss of staining of the matrix in six of the cold perfused knees, and in four of these there was also loss of staining of chondrocytes. There was no correlation between the occurrence of these changes and the time interval between perfusion and examination, the changes being seen after one day (1/3), one week (1/2), two weeks (3/8), and 12 weeks (1/3) (Fig. 3). Loss of staining was seen in the unperfused left knee of one animal seven days after cold silicone treatment to the opposite knee, which itself showed no cartilage abnormality. The articular cartilage was normal in all the other joints examined whether from the perfused or unperfused side (Fig. 3b).

Areas of previous bone necrosis were seen in the subchondral bone in four of the cold perfused knees where there were cartilage changes. There was evidence of healing, with appositional new bone formation and normal fat and haemopoietic tissue in the related intertrabecular spaces (Fig. 4).

\section{Incorporation of radioactive sulphate into cartilage}

Fig. 5 shows the rate of radioactive sulphate incorporation into the cartilage of the cryo-irrigated knees compared with the untreated knees among the 16 rabbits. In none of these animals had arthritis been induced.

All the specimens incorporated sulphate, but there was considerable variation between individual joints. Variation is to be expected in this type of experiment because $(a)$ removal of cartilage slices by scalpel yields material from different depths (incorporation rates vary with depth) and (b) the

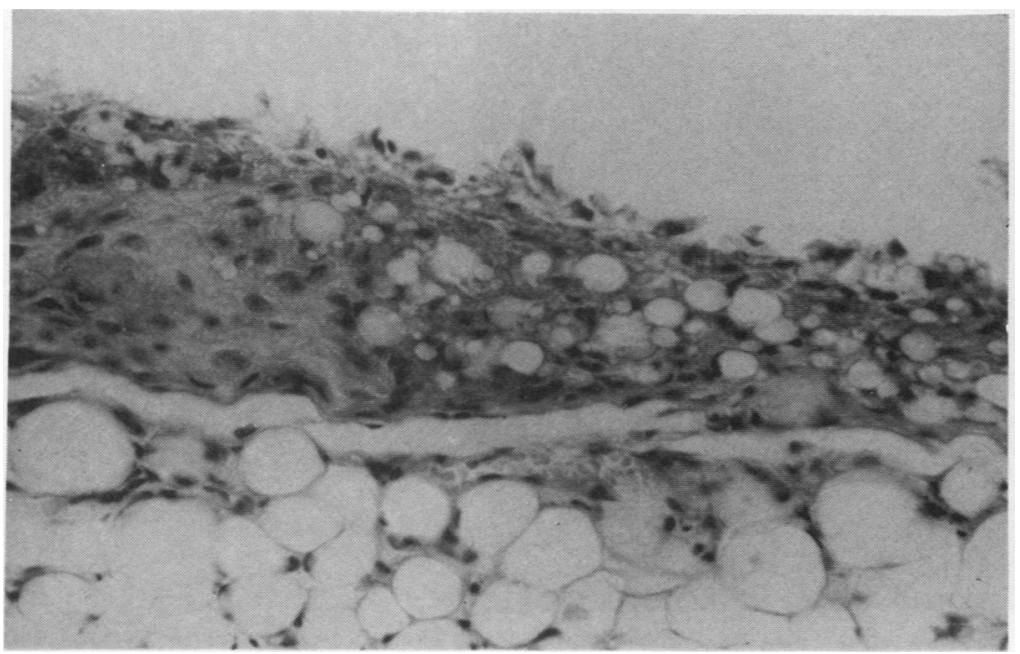

Fig. 2 Synovium one week after perfusion of joint with cold silicone, showing intimal cell hyperplasia and numerous round spaces representing the sites of silicone deposition. (Haematoxylin-eosin). 
washing steps can cause loss of radioactivity. For these reasons the values represent minimum incorporation rates. The mean values were higher for the treated joints (treated, mean 1.34, median 1.30; untreated, mean $1 \cdot 21$, median $1 \cdot 34$ ), indicating that cryo-irrigation had produced no overall interference with sulphate incorporation. The possibility remains, however, that the two lowest incorporation rates of the treated joints were influenced by the cooling procedure.

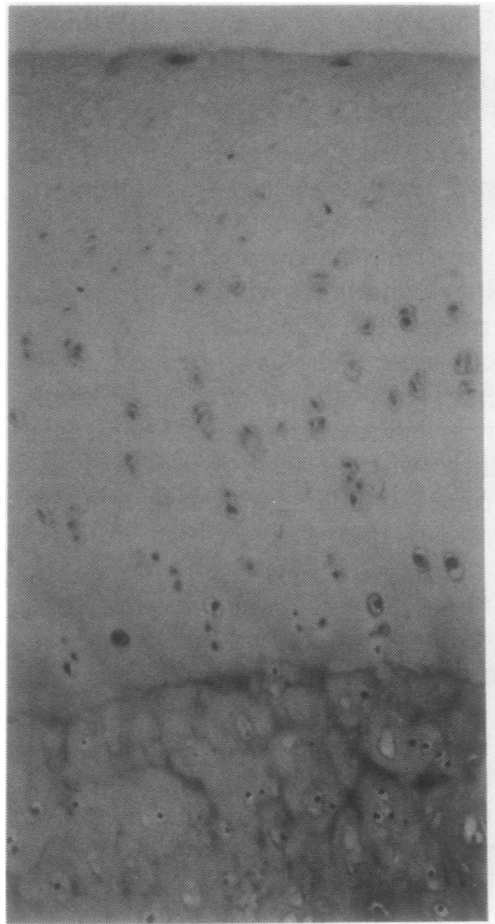

Fig. 3a

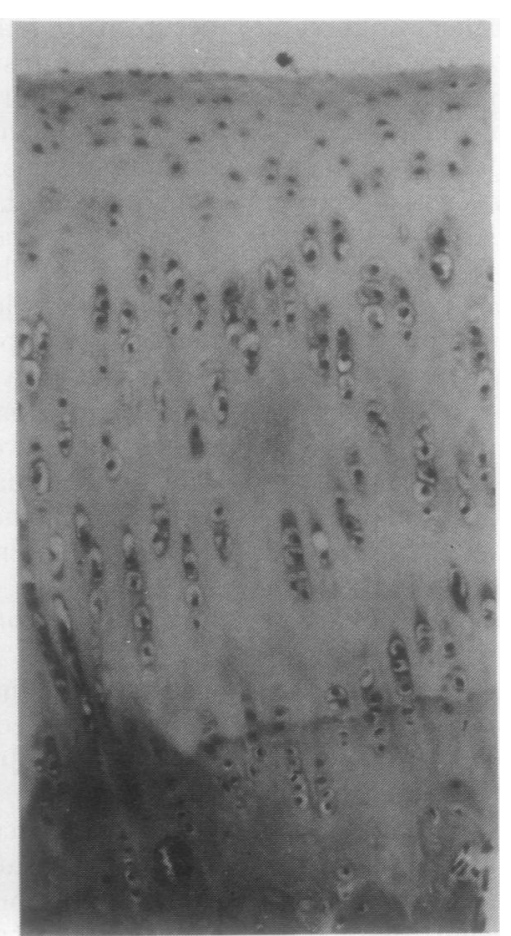

Fig. 3b

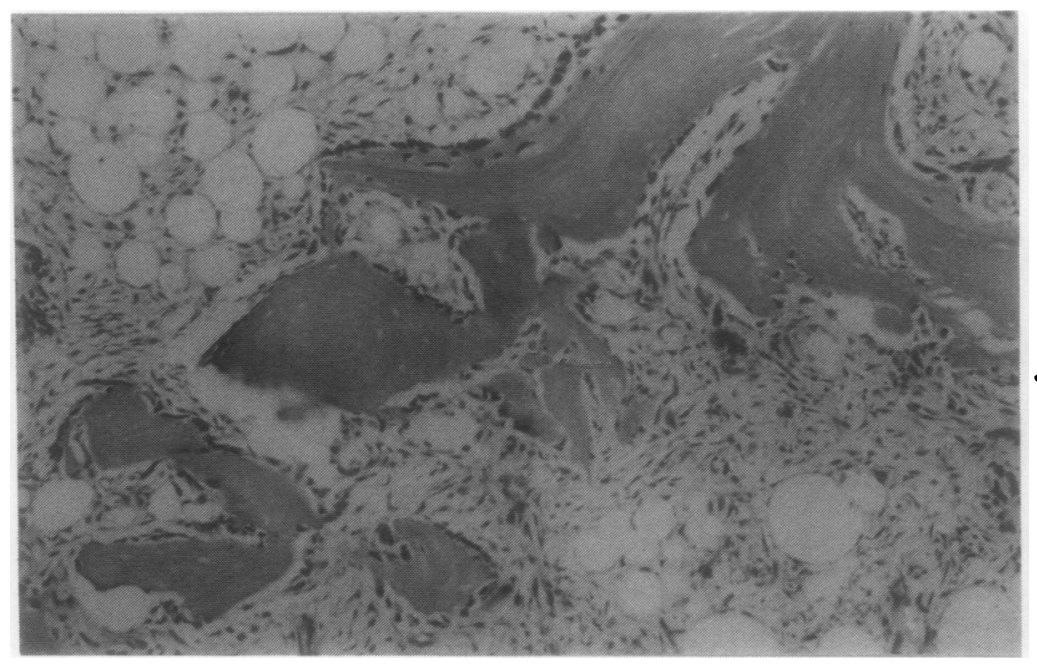

Fig. 3 (a) Articular cartilage two weeks after perfusion of joint with cold silicone, showing loss of staining of chondrocyte nuclei; (b) articular cartilage from the unperfused control joint of the same animal.

(Haematoxylin-eosin).

Fig. 4 Bone necrosis in the subchondral bone, with appositional new bone formation and fibrosis of the fatty marrow, 14 days after cold silicone perfusion of joint. (Haematoxylin-eosin). 
There was no relation between the rate of incorporation of radioactive sulphate and the interval from perfusion to sacrifice of the animals. No correlation was found between loss of staining and the rate of sulphate incorporation (Fig. 5).

\section{NORMAL JOINTS PERFUSED WITH SILICONE} AT AMBIENT TEMPERATURE

Six animals were perfused with silicone of different viscosities at ambient temperature and killed five weeks later. The synovium in all the perfused joints showed appearances like those seen after cold silicone treatment. Loss of articular cartilage staining was seen in both joints treated with $200 / 1 \mathrm{cSt}$ silicone, and one of these showed cell death. Both of

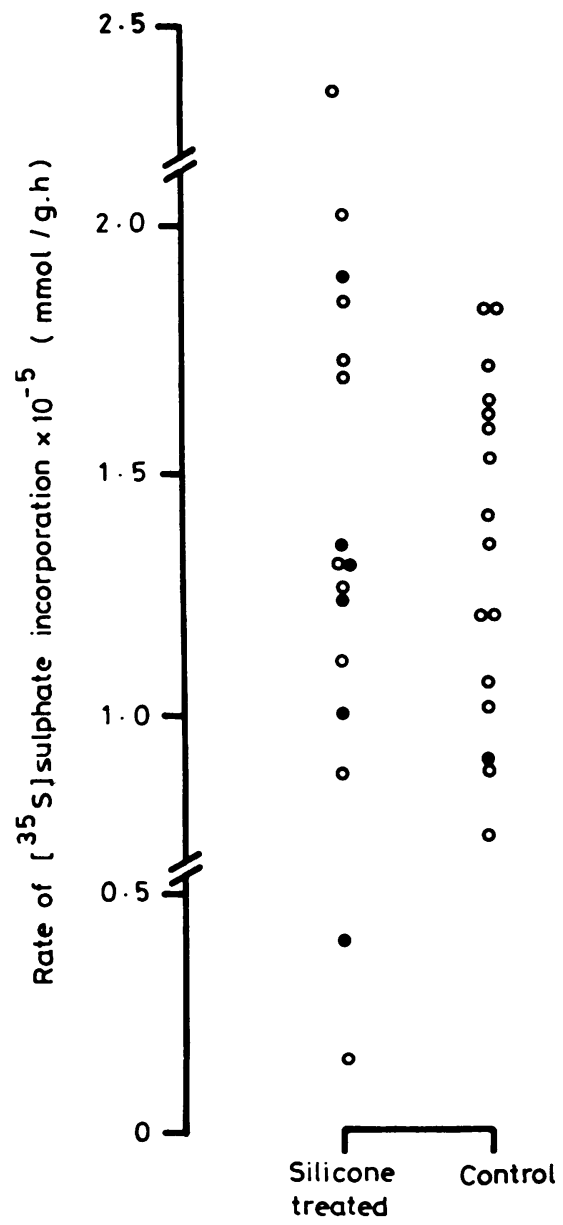

Fig. 5 Rate of $~_{35}^{35}$ Jsulphate incorporation by cartilage from cold silicone treated and control (untreated) rabbit knee joints. Histological appearances of cartilage from the same joints, $\mathrm{O}=$ normal, $\mathrm{O}=$ loss of staining. these joints showed an area of subchondral bone with appositional new bone as evidence of healing. These appearances were similar to those seen with cold perfusion in experiment 1 . Those joints perfused with 200/10 and 200/200 cSt material showed no cartilage or bone changes. No abnormality of the synovium, bone, or cartilage was detected in the contralateral knees in any of the animals.

COLD SILICONE PERFUSION OF JOINTS WITH SYNOVITIS INDUCED BY BSA SENSITISATION Seventeen rabbits sensitised with BSA and challenged into the knee on two occasions were available for examination of the effects of cold silicone perfusion on this experimental synovitis. They were cold perfused with $200 / 10 \mathrm{cSt}$ silicone into one joint five weeks after the second intra-articular challenge. Five were killed after one day, four after one week, seven after two weeks, and one after four weeks.

A well developed synovitis was apparent in the sensitised and challenged joints on the side not receiving cold silicone treatment. This was characterised by intimal cell hyperplasia and surface fibrin deposition. There was a diffuse infiltrate of lymphocytes, and plasma cells and lymphoid follicles were present in three cases after one day, two at one week, and one at two weeks (Figs $6 a$ and $7 a$ ). The typical features of silicone in the tissues were seen in the synovium of the perfused joints (see Fig. 2). One day after cold silicone perfusion there was also some infiltration with lymphocytes and plasma cells (Fig. 6b), while polymorphonuclear leucocytes were seen in two of these synovia. One week after perfusion the synovitis was less well marked compared with the untreated side (Fig. 7b) after perfusion, but lymphocytes and plasma cells were still present. Two weeks after cold treatment no lymphoid infiltration and only a few plasma cells were seen in six out of seven animals. Four weeks after treatment one animal showed mild residual synovitis in the perfused joint, while a further single rabbit showed decreased synovial inflammation. Examination of the cartilage showed some loss of staining of the cartilage matrix in three silicone perfused joints and in three which had not been perfused. In two of these changes were observed in animals killed seven days after cold silicone perfusion and a further example after two weeks, while the unperfused joints showed abnormality in one animal after one day and in two others seven days from the beginning of the experiment.

COLD SILICONE PERFUSION OF A NORMAL DOG KNEE JOINT

The right knee joint of a single dog was perfused 


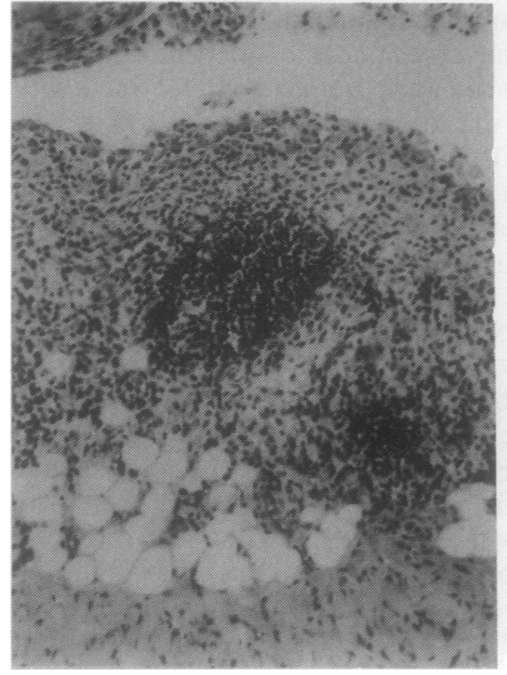

Fig. 6a

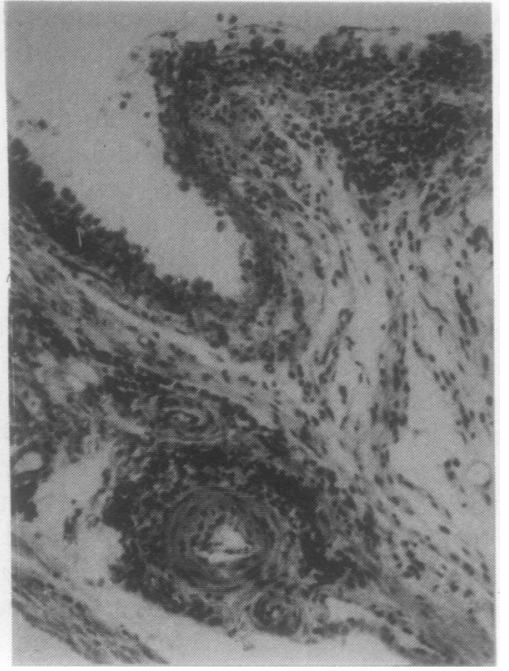

Fig. 6b

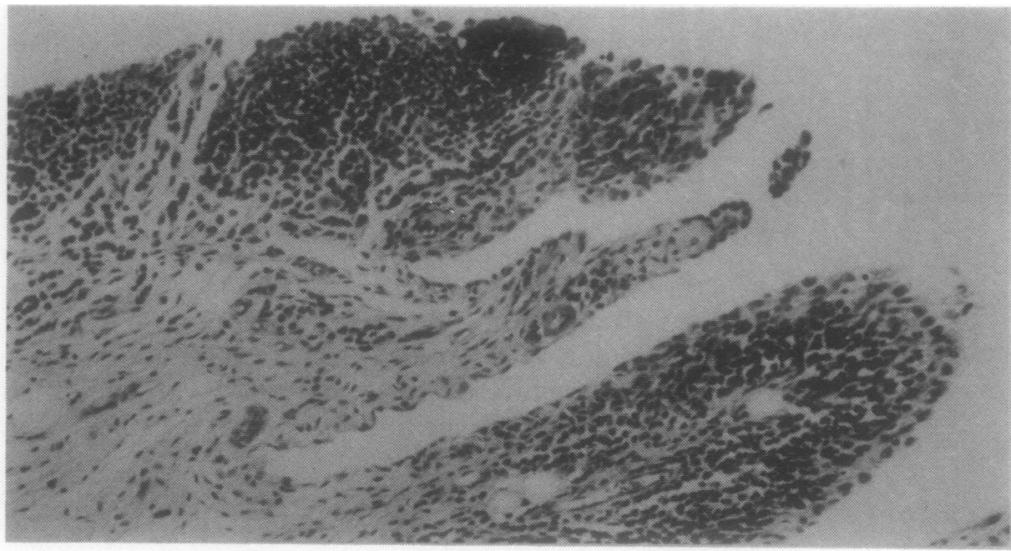

Fig. 7a

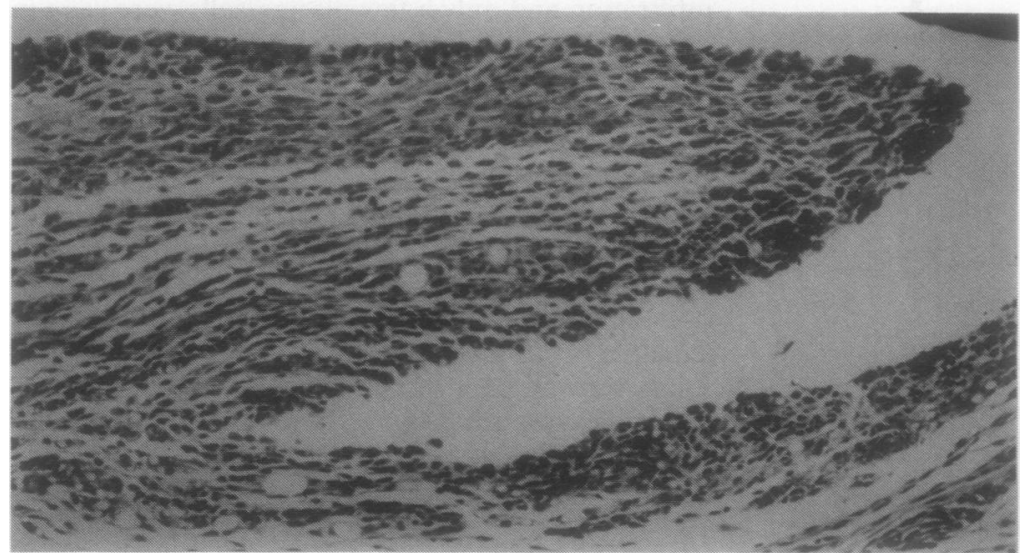

Fig. 7b

Fig. 6 (a) Synovial membrane from the knee of a rabbit sensitised to $B S A$ and five weeks one day after intra-articular challenge with $B S A$. Note the presence of lymphocytes and lymphoid follicles. (b) Synovial membrane from the contralateral knee of the same animal, five weeks one day after BSA challenge and one day after perfusion with cold silicone. Note the small lymphoid follicle and a perivascular collection of lymphocytes.

(Haematoxylin-eosin).

Fig. 7 (a) Synovial membrane from the knee of a rabbit sensitised with BSA examined six weeks after intra-articular challenge with $B S A$, showing well developed synovitis with lymphoid follicles.

(b) Synovial membrane from the contralateral joint of the same animal, six weeks after intra-articular challenge and one week after perfusion with cold silicone. Note decrease in the synovitis with no lymphoid follicles, though there are still lymphocytes present. There is intimal cell hyperplasia and bead-like silicone spaces are present. (Haematoxylin-eosin). 


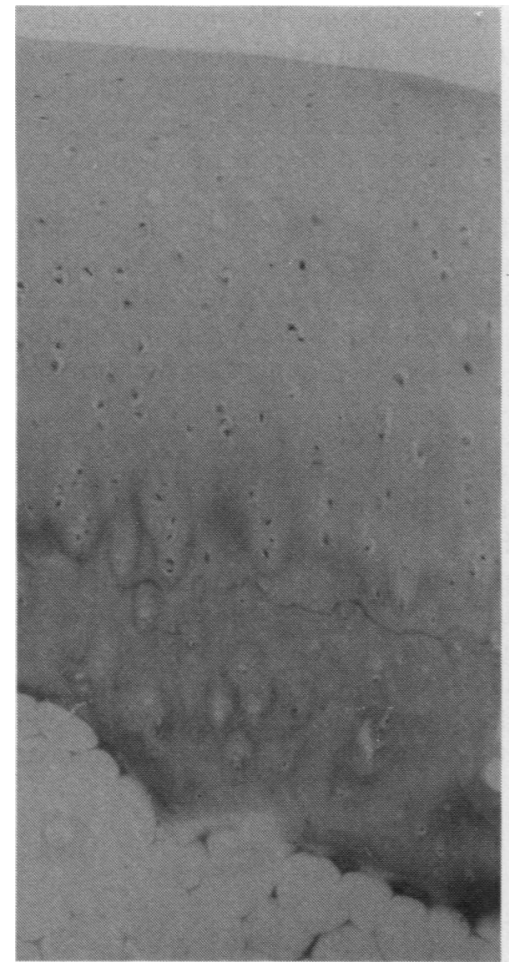

Fig. 8a

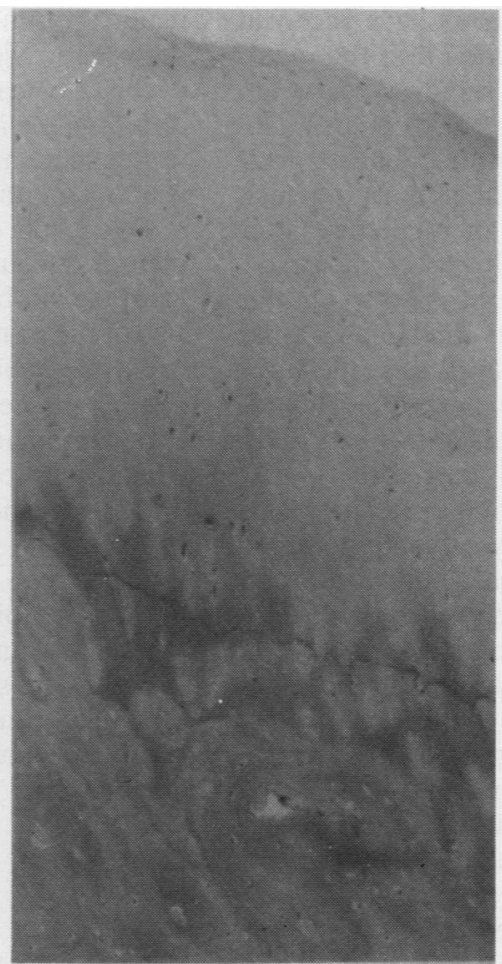

Fig. 8b
Fig. 8 Articular cartilage from (a) control (left) knee of dog and (b) the right knee of the same dog, showing no difference in appearance from normal six months after cold silicone perfusion. (Haematoxylin and eosin). with cold silicone $(200 / 10 \mathrm{cSt})$ at -6 to $-9^{\circ} \mathrm{C}$ for 22 minutes. The animal showed no adverse effects during the procedure and subsequently remained fully mobile. It was killed six months later and both knees examined histologically. No abnormalities were detected in the knees macroscopically and no histological changes were seen in the bone, cartilage, or synovium on either side, apart from the typical appearances of silicone in the soft tissues of the perfused joint. Specifically there was no bone death or loss of cartilage staining (Fig. 8).

\section{Discussion}

The results of these experiments indicate that it is technically feasible to cryo-irrigate the rabbit knee joint, and that such cryo-irrigation produces a significant reduction in experimental immune synovitis. The experience gained in the use of the cooling equipment suggests that comparable cryo-irrigation of the human rheumatoid knee joint would be technically straightforward.

This raises the possibility that cryo-irrigation, if it does not damage cartilage or bone, may offer a useful method of managing the rheumatoid knee with gross synovial proliferation. It has to be accepted that other methods of local synovial ablation have proved disappointing, and neither surgical synovectomy nor radioisotopic or chemical ablation have stood the test of long term controlled trials (though short term pain relief has tended to produce initial enthusiasm). We believe, however, that the possibility of using cryo-irrigation should be pursued because, firstly, these preliminary studies suggest that it may have a favourable influence on experimental synovitis; this is in contrast with the comparable experiments carried out in this laboratory, in which surgical synovectomy was tested in a somewhat similar manner. ${ }^{1}$ Secondly, cryoirrigation avoids the hazards of radioactivity and might thus offer a means of repeated treatments and a means of treating younger patients.

These experiments provide only very preliminary information about the safety of this technique. The injection of silicone itself (at ambient temperature) clearly produces some histological changes in the synovium over the period covered by these experiments. Whether these may be harmful is unknown. 
Certainly, large numbers of patients have received intra-articular injections of silicone (with the material being left in the joint, not removed as would happen after cryo-irrigation), and no significant ill effects have been noted. ${ }^{2} 3$ The unexplained postoperative deaths of nine out of 26 animals in which cryo-irrigation was carried out in one knee after induction of synovitis in both knees leaves a question mark over the general safety of this procedure. Further experiments involving larger animals will be required to answer this question.

Another important question is whether cryoirrigation damages articular cartilage. Six out of 16 cold treated joints (and 1/16 untreated joints) showed some minor loss of cartilage staining. This could indicate altered chondrocyte function, which might be temporary. The sulphate incorporation studies indicate that there was no gross chondrocyte dysfunction in most of the treated joints. The presence of subchondral bone death in some animals is evidence that the blood supply to the bone may be in jeopardy during the cold irrigation procedure. The fact that similar loss of staining and subchondral bone death occurred in animals perfused with silicone at ambient temperature, however, suggests the possibility that the actual perfusion of the joint rather than the temperature of the perfusate may be important. It is possible that despite careful design of the apparatus to avoid high pressure in the joint this occurred in some animals and was the cause of local alteration in blood supply, with damage to subchondral bone. In all animals in which this occurred there was evidence of well established healing around the dead bone. The poor correlation between $\left[{ }^{35} \mathrm{~S}\right]$ sulphate incorporation and the results of histological analysis may be due to sampling differences within the joint. It is difficult, otherwise, to reconcile high sulphate incorporation with loss of staining in the cartilage.

The result of the single longer term dog experiment is encouraging, especially since no evidence of cartilage or bone changes was seen. Whether the size of the joint is important is unknown, and further experiments in the dog are planned to examine this system further.

In summary, these results show that cryoirrigation is feasible in the rabbit knee joint, and the procedure should therefore present no technical problems in the larger human rheumatoid knee joint. It can produce inhibition of experimental immune synovitis, with what may be no more than transient changes in articular cartilage. We believe that these results justify more detailed and longer term experiments in animals, with a view to the possible use of this technique in the diseased human knee joint.

\section{References}

1 Currey H L F, Moore C J, Prentice A I D. Surgica synovectomy and experimental immune synovitis in the rabb knee joint. Ann Rheum Dis 1970; 29: 503-8.

2 Helal B, Karadi B S. Artificial lubrication of joints: use of silicone oil. Ann Phys Med 1968; 9: 334-40.

3 Helal B. Silicones in orthopaedic surgery. In: Apley A G, ed. Recent advances in orthopaedics. London: Churchill Living stone, 1969: 91-114. 